\title{
Unique N-Terminal Interactions Connect F-BOX STRESS INDUCED (FBS) Proteins to a WD40 Repeat-like Protein Pathway in Arabidopsis
}

\author{
Edgar Sepulveda-Garcia ${ }^{1,2}$, Elena C. Fulton ${ }^{3}$, Emily V. Parlan ${ }^{3}$, Lily E. $\mathrm{O}^{\prime}$ Connor $^{3}$, Anneke A. Fleming ${ }^{3}$, \\ Amy J. Replogle ${ }^{3}$ (D), Mario Rocha-Sosa ${ }^{2}$, Joshua M. Gendron ${ }^{4}$ and Bryan Thines ${ }^{3, *}$ \\ 1 Instituto de Biotecnología, Universidad del Papaloapan, Tuxtepec 68301, Mexico; esepulveda@unpa.edu.mx \\ 2 Departamento de Biología Molecular de Plantas, Instituto de Biotecnología, Universidad Nacional Autónoma \\ de México, Cuernavaca 62250, Mexico; rocha@ibt.unam.mx \\ 3 Biology Department, University of Puget Sound, Tacoma, WA 98416, USA; \\ efulton@alumni.pugetsound.edu (E.C.F.); eparlan@alumni.pugetsound.edu (E.V.P.); \\ l.e.oconnor@wustl.edu (L.E.O.); afleming@alumni.pugetsound.edu (A.A.F.); \\ areplogle@pugetsound.edu (A.J.R.) \\ 4 Department of Molecular, Cellular and Developmental Biology, Yale University, New Haven, CT 06511, USA; \\ joshua.gendron@yale.edu \\ * Correspondence: bthines@pugetsound.edu
}

check for updates

Citation: Sepulveda-Garcia, E.; Fulton, E.C.; Parlan, E.V.; O'Connor, L.E.; Fleming, A.A.; Replogle, A.J.; Rocha-Sosa, M.; Gendron, J.M.; Thines, B. Unique N-Terminal Interactions Connect F-BOX STRESS INDUCED (FBS) Proteins to a WD40 Repeat-like Protein Pathway in Arabidopsis. Plants 2021, 10, 2228. https:// doi.org/10.3390/plants10102228

Academic Editors: Ewa Muszyńska, Kinga Dziurka and Mateusz Labudda

Received: 16 September 2021

Accepted: 11 October 2021

Published: 19 October 2021

Publisher's Note: MDPI stays neutral with regard to jurisdictional claims in published maps and institutional affiliations.

Copyright: () 2021 by the authors. Licensee MDPI, Basel, Switzerland. This article is an open access article distributed under the terms and conditions of the Creative Commons Attribution (CC BY) license (https:// creativecommons.org/licenses/by/ $4.0 /)$.
Abstract: SCF-type E3 ubiquitin ligases provide specificity to numerous selective protein degradation events in plants, including those that enable survival under environmental stress. SCF complexes use F-box (FBX) proteins as interchangeable substrate adaptors to recruit protein targets for ubiquitylation. FBX proteins almost universally have structure with two domains: A conserved N-terminal F-box domain interacts with a SKP protein and connects the FBX protein to the core SCF complex, while a C-terminal domain interacts with the protein target and facilitates recruitment. The F-BOX STRESS INDUCED (FBS) subfamily of plant FBX proteins has an atypical structure, however, with a centrally located F-box domain and additional conserved regions at both the N- and C-termini. FBS proteins have been linked to environmental stress networks, but no ubiquitylation target(s) or biological function has been established for this subfamily. We have identified two WD40 repeat-like proteins in Arabidopsis that are highly conserved in plants and interact with FBS proteins, which we have named FBS INTERACTING PROTEINs (FBIPs). FBIPs interact exclusively with the N-terminus of FBS proteins, and this interaction occurs in the nucleus. FBS1 destabilizes FBIP1, consistent with FBIPs being ubiquitylation targets $\mathrm{SCF}^{\mathrm{FBS} 1}$ complexes. This work indicates that FBS proteins may function in stress-responsive nuclear events, and it identifies two WD40 repeat-like proteins as new tools with which to probe how an atypical SCF complex, $\mathrm{SCF}^{\mathrm{FBS}}$, functions via FBX protein N-terminal interaction events.

Keywords: F-box protein; SCF complex; stress response; WD40 repeat-like protein

\section{Introduction}

At the onset of environmental stress, the ubiquitin 26S proteasome system (UPS) selectively degrades key cellular proteins to initiate plant responses that promote resilience and survival. Protein targets destined for removal are ubiquitylation substrates for E3 ubiquitin ligases, where one prevalent E3 ligase subtype is the SKP1-CUL1-F-box (SCF) complex [1]. SCF complexes use an interchangeable F-box (FBX) protein subunit as a substrate adaptor to specifically interact with unique protein targets [2-5]. FBX proteins almost universally have a structure with two domains: An N-terminal F-box domain facilitates interaction with a SKP protein and the core SCF complex, and a C-terminal domain interacts specifically with the target(s) [2]. This two-domain structure directly bridges core UPS components to precise protein targets under specific conditions, and it 
places FBX proteins at a dynamic interface that regulates diverse cellular pathways critical for plant life.

A very small number of FBX proteins, however, deviate from this typical two-domain protein structure. Many of these atypical FBX proteins have a centrally located F-box domain, a C-terminal target interaction domain, and an additional protein interaction domain at the N-terminus [6-8]. In humans, $\mathrm{N}$-terminal domains can control subcellular localization [9], bind to an accessory protein that assists with C-terminal targeting events [10] or mediate regulatory interactions with other proteins $[6,11,12]$. The only plant FBX proteins with established N-terminal interaction dynamics belong to the ZEITLUPE (ZTL), FLAVIN-BINDING KELCH REPEAT F-BOX1 (FKF1), and LOV KELCH PROTEIN2 (LKP2) subfamily, which regulate the circadian clock and flowering time [8,13-16]. In addition to a central F-box domain, the ZTL/FKF1/LKP2 subfamily has a N-terminal blue-light sensing LOV domain and C-terminal kelch repeats [16], which are both used to recruit distinct ubiquitylation substrates $[8,15,17,18]$. The N-terminal LOV domain has additional roles that regulate the FBX function through an interaction with GIGANTEA (GI), which controls subcellular localization and protein stability $[13,14]$. Therefore, across kingdoms, a few atypical FBX proteins with a N-terminal protein interaction domain, in addition to a C-terminal targeting domain, achieve an expanded function by having further regulatory capacity and/or coordinating multiple cellular outputs through dual targeting.

F-BOX STRESS INDUCED (FBS) proteins constitute a far less understood subfamily of plant FBX proteins with an atypical structure [19-21]. Arabidopsis FBS1 is the founding member of this subfamily and is noteworthy for its broad biotic and abiotic stress-inducible gene expression profiles [19,21]. In FBS1, a centrally located F-box domain is flanked by two conserved regions present at the $\mathrm{N}$ - and C-termini, which do not match any known protein interaction domains or motifs [19]. FBS1 interacts with Arabidopsis SKP1 (ASK1) and can auto-ubiquitylate $[19,20]$, suggesting that it forms a functional SCF-type E3 ligase in vivo. At least five of 13 Arabidopsis 14-3-3 regulatory proteins bind to FBS1 [20]. However, since this interaction requires both the N-terminal region and the F-box domain of FBS1 [20], and ubiquitylation presumably requires an unhindered F-box domain to interact with the SKP subunit of the SCF complex [1], the 14-3-3 proteins are unlikely ubiquitylation targets. Furthermore, an inducible FBS1 gene construct had no discernable effect on FBS1 interactor 14-3-3 $\lambda$ protein abundance [20]. Importantly though, FBS1-interacting 14-3-3 proteins are negative regulators of Arabidopsis responses to cold and salt stress [22-26], which demonstrates another important link between FBS1 and environmental stress response networks in plant cells.

A more complete understanding of the FBS family protein function in plants has been stymied by two primary limitations. First, not knowing selective targeting relationships between $\mathrm{SCF}^{\mathrm{FBS}}$ complexes and their putative substrates has left FBS action on cellular output pathways completely enigmatic. Second, functional redundancy within this family has likely thwarted past efforts seeking to establish a biological function. Arabidopsis fbs1 plants have no obvious phenotype [19,21], however, three additional FBS family members that may be functionally redundant are encoded in the genome. Here, we identify two highly conserved WD40 repeat-like proteins that interact with multiple FBS family members in Arabidopsis, which we have named FBS INTERACTING PROTEINs (FBIPs). Interactions between all four FBS subfamily members and FBIP proteins occur in the nucleus, and interactions occur exclusively via the N-terminal domain of FBS proteins. These findings connect a stress network involving FBS proteins to nuclear processes, and they provide new tools with which to probe unique $\mathrm{N}$-terminal interactions in FBX proteins in the context of plant stress responses.

\section{Results}

\subsection{FBS Protein Interaction with ASK1}

FBS1 is the founding member of a four-member FBX protein subfamily (FBS1-FBS4) in Arabidopsis. FBS2-FBS4, similar to FBS1, share a non-canonical structure with a centrally 
located F-box domain and conserved regions at their N- and C-termini (Figure 1A). The conserved region at the $\mathrm{N}$-termini of FBS proteins spans approximately 20 residues, while the conserved region at the C-terminus encompasses about 35 residues (Figure 1A). FBS1 interacts with ASK1 and auto-ubiquitylates, indicating that FBS1 likely participates in functional SCF complexes $[19,20]$. However, the ability of other FBS family members to interact with ASK proteins remains unknown, as does the possibility of functional redundancy among family members. To interrogate this possibility, all four FBS family members were tested as bait constructs (DBD, GAL4 DNA-binding domain) for the interaction with ASK1 as prey (AD, GAL4 activation domain) under less stringent (-TLH) and more stringent (-TLHA) nutritional selection. Interactions were apparent between all four FBS family members on -TLH, although only very minimal growth was observed for FBS2 (Figure 1B). Only the interactions between FBS1 and FBS4 with ASK1 were apparent under the most stringent selection (-TLHA) (Figure 1B). Since Arabidopsis has 21 ASK proteins, it is possible that the FBS proteins showing minimal partnering with ASK1 interact more strongly with the other untested ASKs [27]. These interactions show, however, that FBS2-FBS4 are viable candidates for functional SCF complex substrate adapters, similar to FBS1.

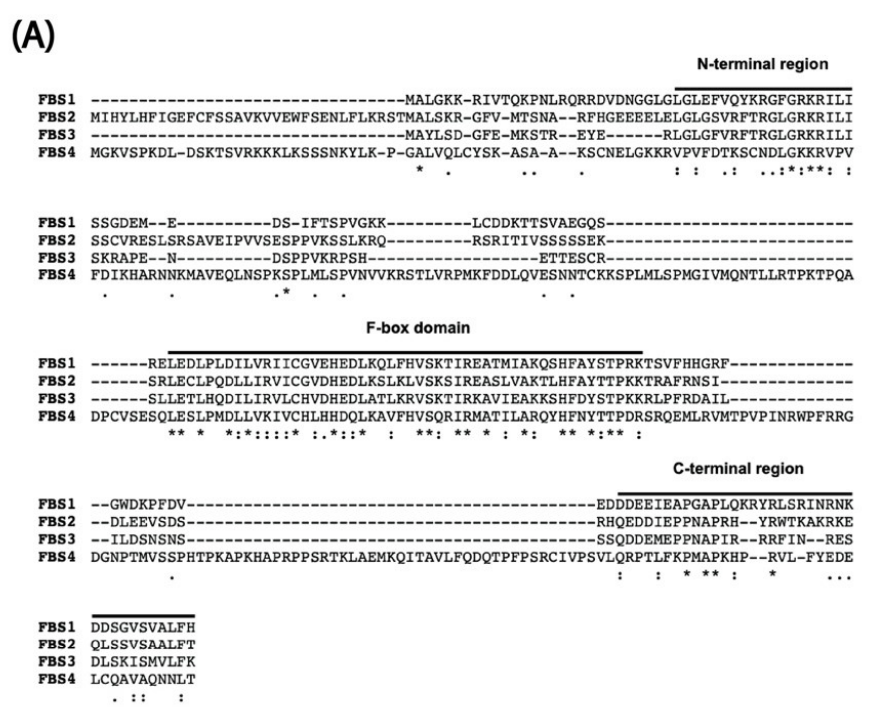

(B)

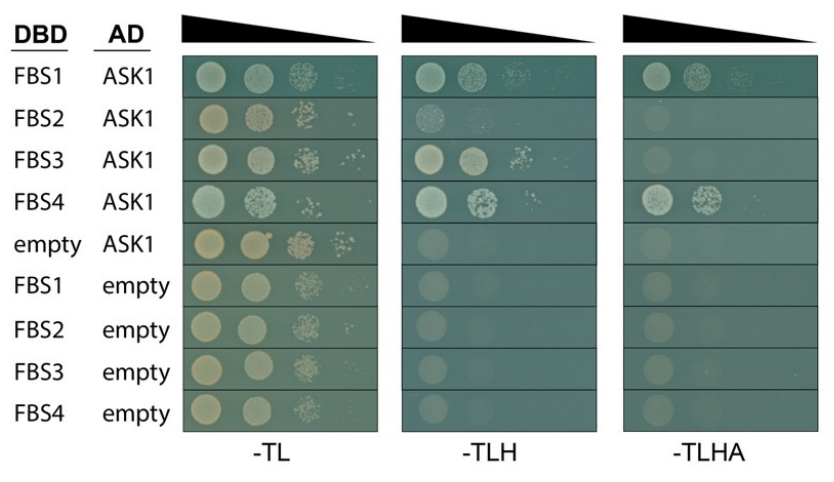

Figure 1. The Arabidopsis F-BOX STRESS INDUCED (FBS) protein family. (A) Full-length protein sequence alignment of the four Arabidopsis FBS family members (FBS1-FBS4) created with the T-COFFEE sequence alignment program. Asterisks are fully conserved residues, colons are strongly conserved residue properties, and periods are weakly conserved residue properties. (B) FBS family interactions with ASK1 in yeast two-hybrid assays. Diploid yeast strains with indicated test constructs as bait (DBD) and prey (AD) were grown in liquid culture, diluted $\left(\mathrm{OD}_{600}=10^{0}, 10^{-1}, 10^{-2}\right.$, $10^{-3}$ ), and spotted on SD medium minus Trp/Leu (-TL), minus Trp/Leu/His (-TLH), and minus Trp/Leu/His/Ade (-TLHA). 


\subsection{Identification of a New FBS1 Interactor}

In addition to ASK1, the only established FBS1 interacting proteins belong to the 14-3-3 family [20]. However, since the interaction dynamics are not consistent with ubiquitylation of 14-3-3 proteins by $\mathrm{SCF}^{\mathrm{FBS} 1}$ [20], we sought additional FBS1 interactors as candidate targets that could connect FBS proteins to biological processes. Two additional related proteins were identified as partners for FBS1, which we have named FBS INTERACTING PROTEINs (FBIPs). FBIP1 (At3g54190) was identified in the same yeast two-hybrid screen that found 14-3-3 proteins as FBS1 interactors [20]. FBIP1 is also listed as an FBS1 interactor by the SUBA4 database (http: / / suba.live/, accessed on 16 September 2021) from high-throughput protein-protein interaction (PPI) screening $[28,29]$. FBIP1 is 467 residues in length and is a member of the transducin/WD40 repeat-like superfamily of proteins. WD40 repeats typically form a $\beta$-propeller domain that acts as a scaffold in mediating protein-protein or protein-DNA interactions [30]. Seven putative WD40 repeat-like sequences were predicted in FBIP1 by the WD40-repeat protein Structures Predictor database version 2.0 (WDSPdb 2.0) [31], although these predictions fall into the low confidence category (Figure 2). A second protein highly similar to FBIP1 was identified in the Arabidopsis genome by BLAST search, which we have named FBIP2 (At2g38630). The protein sequence identity and similarity between FBIP1 and FBIP2 are just over 91\% and $96 \%$, respectively (Figure 2 ).

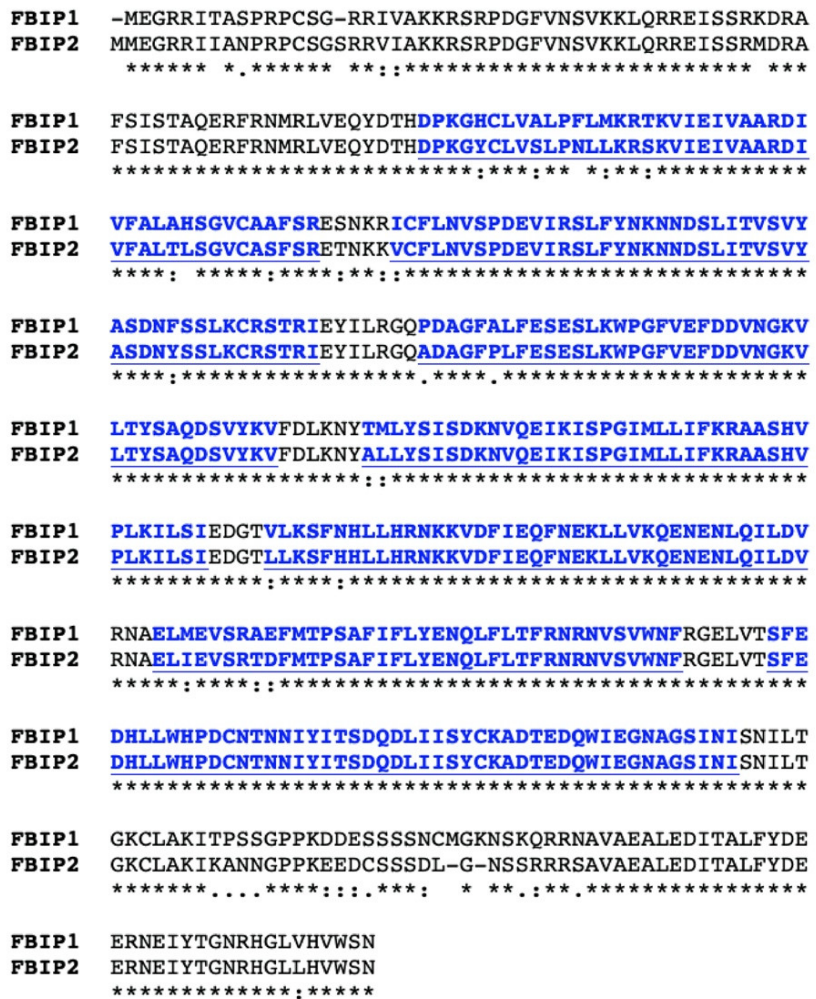

Figure 2. FBS INTERACTING PROTEIN (FBIP) sequence features. Full-length protein sequence alignment of the two Arabidopsis FBIP family members created with the T-COFFEE sequence alignment program. Blue indicates locations of seven WD40-like repeat sequences predicted by the WD40-repeat protein Structure Predictor version 2.0 (WDSPdb 2.0). Asterisks are fully conserved residues, colons are strongly conserved residue properties, and periods are weakly conserved residue properties.

We gained no additional insight on the FBIP function using various bioinformatics resources. Other than putative WD repeat-like sequences, no sequence features were identified using various domain or motif prediction programs. BLAST and PSI-BLAST searches with FBIP1 and FBIP2 sequences failed to identify additional significant hits in Arabidopsis. We did, however, find very highly conserved FBIP protein sequences 
throughout the plant kingdom, including in bryophytes (the top BLAST hit in Physcomitrella patens is about $77 \%$ identical and $85 \%$ similar to Arabidopsis FBIP1). By investigating AtGenExpress ATH1 array datasets [32-34], we found that FBIP1 is constitutively expressed in most tissues and organs of Arabidopsis, and throughout its life cycle, but we found no conditions where FBIP1 is more highly expressed compared to the other conditions. FBIP2 is not represented on the ATH1 array.

\subsection{FBS Interactions with FBIPS}

We confirmed that the full-length FBS1 and FBIP1 interact with yeast two-hybrid analysis. The interaction between FBS1 and FBIP1 elicited growth in yeast strains on both less stringent (-TLH) and more stringent (-TLHA) nutritional selection, and FBS1 yielded growth with FBIP2 on -TLH (Figure 3A). Family-wide interactions between each FBS protein and the two FBIP proteins were also assessed (Figure S1). Growth was observed for FBS3 and FBIP1, but not with FBS2 or FBS4. No additional interactions were observed with FBIP2. Collectively, the yeast two-hybrid results suggest that FBS1 and FBIP1 might be the primary FBS/FBIP protein interaction pair or possibly bind with the strongest affinity, but that some other family-wide interactions might be possible.

(A)

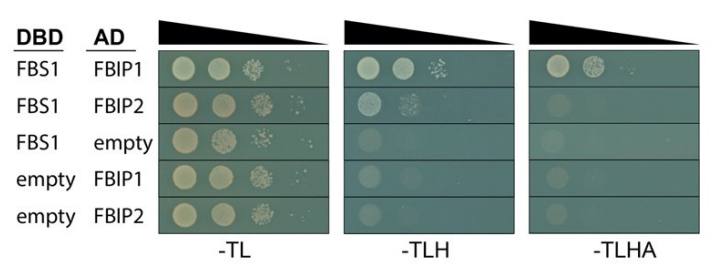

(B)
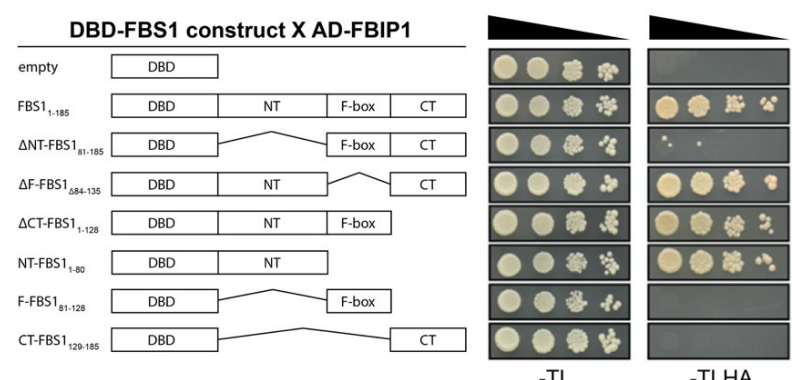

Figure 3. Yeast two-hybrid ( $\mathrm{Y} 2 \mathrm{H})$ interactions between FBS1 and FBIP proteins. (A) Full-length FBS1 interactions with full-length FBIP1 and FBIP2. Diploid yeast strains with indicated test constructs as bait (DBD) and prey (AD) were grown in liquid culture, diluted $\left(\mathrm{OD}_{600}=10^{0}, 10^{-1}, 10^{-2}\right.$, $10^{-3}$ ), and spotted on SD medium minus Trp/Leu (-TL), minus Trp/Leu/His (-TLH), and minus Trp/Leu/His/Ade (-TLHA). (B) Truncated FBS1 bait (DBD) construct interaction with full length FBIP1 prey (AD). Amino acid deletions are indicated on the left.

FBS proteins have two regions of unknown function outside of the F-box domain and, presumably, at least one of these interacts with a target. In order to determine which parts of FBS1 are important for the FBIP1 interaction, we created truncated versions of FBS1 with the N-terminal (NT), F-box or C-terminal (CT) regions removed in different combinations and tested under stringent (-TLHA) selection (Figure 3B). Removing the $\mathrm{N}$-terminal region ( $\left.\triangle \mathrm{NT}-\mathrm{FBS1}_{81-185}\right)$ abolished the ability of FBS1 to interact with FBIP1, while removal of the F-box domain $\left(\Delta \mathrm{F}-\mathrm{FBS1}_{\Delta 84-135}\right)$ or C-terminal region $\left(\Delta \mathrm{CT}-\mathrm{FBS1} 1_{1-128}\right)$ did not. The FBS1 N-terminal region (NT-FBS1 $1-80$ ) in combination with the full-length FBIP1 yielded growth on -TLHA, indicating that the FBS1 N-terminal domain alone is sufficient to mediate this interaction. 
Near the conserved N-terminal domains of FBS1 and FBS2 we found a LXLXL sequence (Figure 1A), which is the most prominent form of an EAR motif found in many different types of transcriptional regulators [35,36]. The EAR motif mediates the interaction with the WD40 repeat-containing protein TOPLESS (TPL) and TOPLESS RELATED (TPR) co-repressor proteins [37-39]. We considered whether this LXLXL sequence in the N-terminal region of FBS1 might: (1) Function as a canonical EAR motif to interact with TOPLESS, and/or (2) if it could be important for mediating interactions with FBIPs. However, substituting all three leucine residues for alanine in FBS1 did not alter its interaction with FBIP1, and FBS1 did not interact with TPL (both as bait or as prey) in our yeast two-hybrid system.

\subsection{FBS Interactions with FBIP Occur in the Nucleus}

Next, we used bimolecular fluorescence complementation (BiFC) to test the FBS interaction with FBIP in plants and determine where the interaction occurs in a cell. The FBS and FBIP family proteins were expressed in Nicotiana benthamiana leaves as C-terminal fusions to either N-terminal (nYFP) or C-terminal (cYFP) halves of yellow fluorescent protein (YFP). In multiple independent experiments, the YFP fluorescence was observed for pairings between FBS1 and FBIP1 and FBIP2 (Figure 4). This YFP signal co-localized with that of a co-infiltrated H2B-RFP construct, which localizes exclusively in the nucleus [40], and shows that interactions between FBS1 and FBIP proteins also occur in the nucleus. Similar experiments found that FBS2-FBS4 also interact with FBIP1 in the nucleus (Figure S2). We observed interactions for FBS3 and FBS4 with FBIP2 (Figure S3), although we note that these interactions were more variable in the number of YFP positive nuclei across independent replicates, and with consistently fewer interactions for FBS3 and FBIP2. We did not observe any interactions between FBS2 and FBIP2. All FBS and FBIP fusion protein constructs were tested as pairs with empty nYFP or cYFP vectors, and in all pairings we were unable to detect any fluorescent signal similar to the FBS/FBIP test pairs (Figure S4). These findings show that in plants the FBS proteins participate in family-wide interactions in the nucleus.

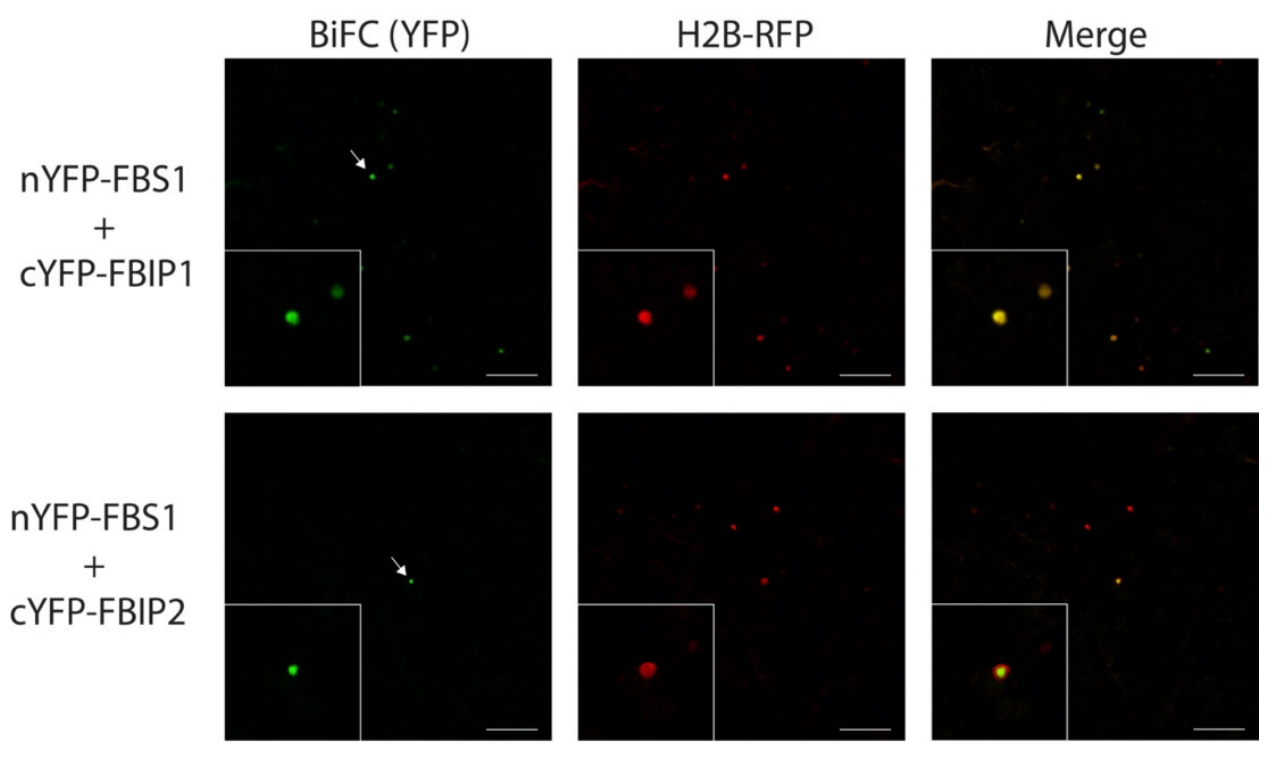

Figure 4. Bimolecular fluorescence complementation (BiFC) interactions between FBS1 and FBIP proteins. Laser-scanning confocal microscopy of $N$. benthamiana epidermal cells expressing $N$-terminal nYFP- or cYFP-tagged FBS1 and FBIP proteins. FBS1 interactions with FBIP1 (top row) or FBIP2 (bottom row) are visualized on the BiFC yellow channel (YFP, left column). A co-expressed H2B-RFP (as nuclear marker) is visualized on the red channel (RFP, middle column) and YFP/RFP images are overlaid (Merge, right column). Arrow indicates selected nuclei in the expanded inset image. Scale bar $=100 \mu \mathrm{m}$. 


\subsection{FBS1 Destabilizes FBIP1}

With the interaction established between multiple FBS and FBIP protein pairs, we next asked if the protein abundance relationship between FBS1 and FBIP1 is consistent with FBIP1 being a ubiquitylation target of $\mathrm{SCF}^{\mathrm{FBS}}$. If a protein is ubiquitylated by a particular SCF complex and subsequently degraded by the $26 \mathrm{~S}$ proteasome, then increasing the abundance of the F-box component typically increases in vivo targeting and decreases substrate abundance [41]. Therefore, we tested the effects of varying FBS1 protein levels on the FBIP1 abundance in our $N$. benthamiana expression system by co-infiltrating Agrobacterium harboring these test constructs in different relative concentrations. Increasing the presence of FBS1 protein resulted in a corresponding decrease in the FBIP1 protein abundance by Western blot analysis (Figure 5). In comparison, when the FBS1 abundance was increased relative to the co-infiltrated $14-3-3 \lambda$ in an identical setup, we did not observe any decrease in 14-3-3 $\lambda$ abundance as the amount of expressed FBS1 was increased (Figure S5). This finding is congruous with previous observations that FBS1 and 14-3-3 interactions are not consistent with targeting [20]. Therefore, since the abundance of FBIP1 decreases in an FBS1-dependent manner, we conclude that FBIPs are viable candidates for $\mathrm{SCF}^{\mathrm{FBS} 1}$ ubiquitylation targets.

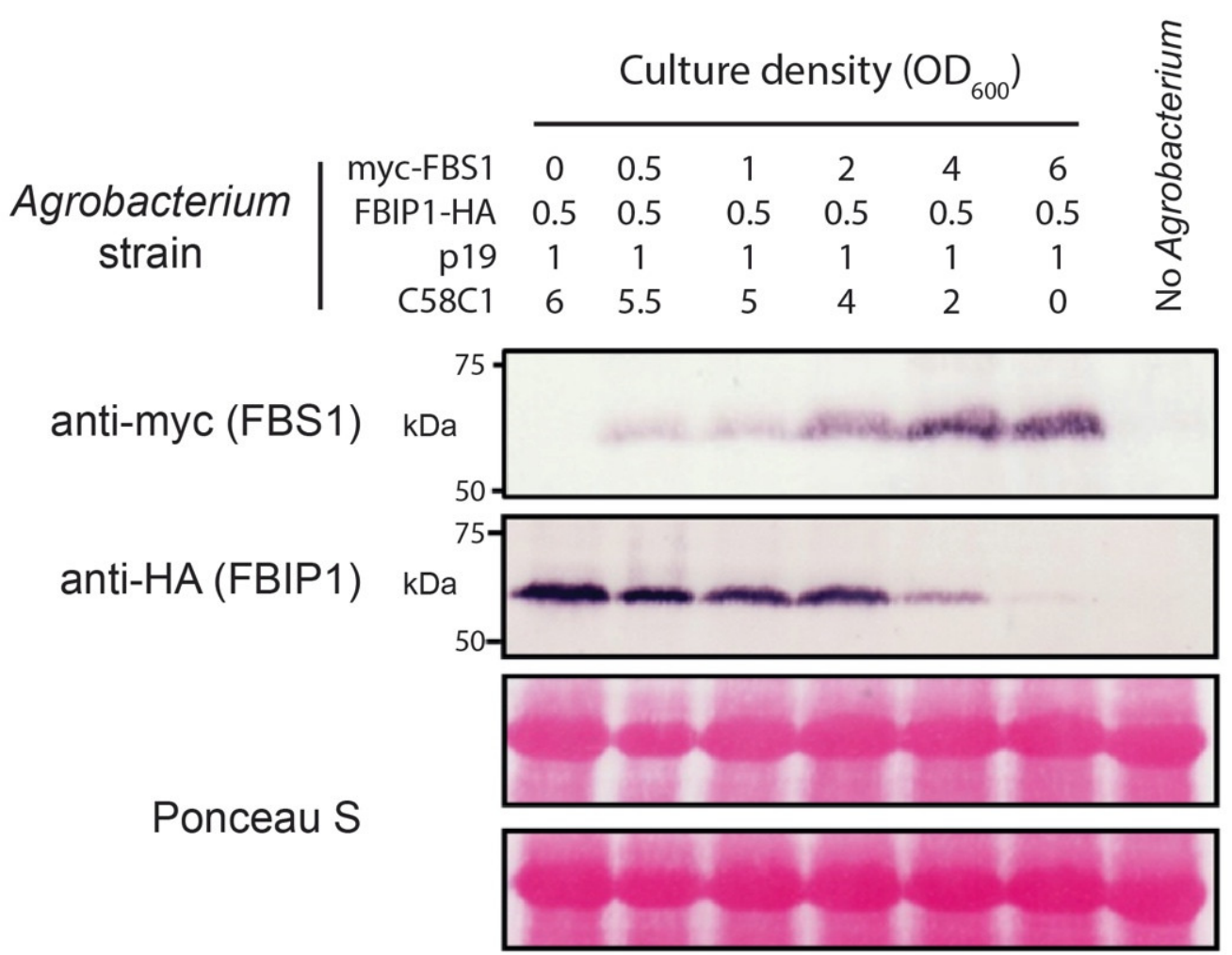

Figure 5. FBS1 influence on FBIP1 protein abundance in plants. N. benthamiana leaves were infiltrated with Agrobacterium (C58C1) strains to express the tagged proteins. Agrobacterium mixes contained varying cell densities of strains harboring expression constructs (myc-FBS1 and/or FBIP1-HA), a suppressor protein (p19) or untransformed cells. Total protein was isolated from leaves 3 days after infiltration, separated by SDS-PAGE, transferred, and probed with antibodies against myc (top row, FBS1) or HA (second row, FBIP1). Bottom two rows show Ponceau S staining of the major subunit of Rubisco from the same two blots as a loading control.

\section{Discussion}

As substrate adapters for SCF-type E3 ligases, FBX proteins act at the interface between core UPS components and specific cellular outputs, including those that help plant cells mitigate the effects of environmental stress. Previous work with FBS1 strongly alluded to some role in plant stress responses, possibly by regulating the expression of stress 
genes [19-21], but a more detailed understanding was limited by the unknown identity of ubiquitylation target(s) and by possible redundancy within the FBS gene family. Here, we have identified a pair of WD40 repeat-like superfamily proteins, FBIP1 and FBIP2, that both interact with FBS family proteins. These family-wide interactions indicate that functional redundancy within these two families is likely, but at the same time suggest a more robust stress response module. The FBS protein interaction with FBIPs in the nucleus points to a role for these proteins in the regulation of gene expression and/or other chromosomal events. Finally, FBIP proteins are strong candidates for $\mathrm{SCF}^{\mathrm{FBS}}$ ubiquitylation targets that act in plant stress responses, and they provide new tools with which to investigate unique FBX protein N-terminal events in plants.

The exclusive nuclear localization FBS and FBIP protein interactions under the conditions we tested offer a critical clue as to the molecular functions of both protein families. One hypothesis for the FBIP function stemming from this result is that they regulate gene expression, which is an idea supported by the finding that hundreds of JA/ABA and other stress genes are mis-expressed in the fbs1-1 background [21]. Some plant nuclear localized WD40 repeat proteins have direct actions in transcription regulation $[39,42,43]$ or chromatin modification [44-46], and in these cases the WD40 repeat proteins are essential components of multi-protein assemblies. For example, TOPLESS (TPL) is a wellstudied WD40 repeat-containing co-repressor protein that acts in diverse developmental and environmental-response pathways [39]. TPL interacts with different DNA-bound transcriptional complexes and it recruits chromatin modifying enzymes and/or Mediator to repress gene expression $[40,47,48]$. TRANSPARENT TESTA GLABRA 1 (TTG1), another WD40 repeat protein, serves as a scaffold and mediates different combinations of bHLH and R2R3-type MYB DNA-binding transcription factors to regulate flavonoid metabolism and various developmental processes $[43,49]$. The FBIP proteins may function similarly to TPL or TTG1 and act as scaffolds and/or in recruitment roles for complexes that regulate transcription. Knowing additional FBIP interactors, which may include more recognizable proteins with readily inferred functions, will help address this hypothesis.

Future work will also be guided by questions that address interaction dynamics between FBIPs and the N-terminal region of FBS proteins, and the consequences of these associations. There are 13 residue positions in the FBS N-terminal region, ranging from moderately to absolutely conserved, that could be critical for the interaction with FBIPs. Future work will include identification of the exact residue or residues in FBS proteins mediating this interaction. Given numerous FBS connections to stress, but that FBIP1 appears to be constitutively expressed across different plant organs and environmental conditions, it could be the case that FBIP proteins are components of a stress-response system working at the post-translational level. Next steps include a rigorous assessment of conditions under which $\mathrm{SCF}^{\mathrm{FBS}}$ complexes form and interact with FBIP proteins in vivo. Furthermore, whether some additional factor (i.e., post-translational modification) stimulates $\mathrm{SCF}^{\mathrm{FBS}}$ association with FBIP proteins, as in the case of some other SCF targeting events [50], is well worth investigating. The idea that additional in vivo factors or modification mediates the FBS/FBIP interaction is consistent with the finding that we observed more family-wide interactions in plant BiFC experiments compared to yeast two-hybrid. Knowing that SCF complexes in some atypical contexts ubiquitylate targets via the FBX protein N-terminal interactions $[8,17]$, and that FBS1 appears to destabilize FBIP1, a leading hypothesis for future work is that FBIP proteins are bona fide ubiquitylation substrates for $\mathrm{SCF}^{\mathrm{FBS}}$. Considering our work here and the general knowledge surrounding SCF action, our current model is that stress stimulates increased $\mathrm{SCF}^{\mathrm{FBS}}$-dependent ubiquitylation of FBIP proteins, which are then degraded in response to this environmental trigger, resulting in cellular changes.

The atypical structure of FBS proteins, along with the identification of FBIPs as FBS Nterminal interactors, leads to a few intriguing hypotheses regarding how this SCF complex may impact cellular pathways in plant stress. If FBIP is a bona fide target with a biological function distinct from a more typical C-terminal target, then $\mathrm{SCF}^{\mathrm{FBS}}$ complexes provide an exciting opportunity to study how plants coordinate more than one cellular pathway 
related to stress. $\mathrm{N}$ - and C-terminal targeting events might be simultaneous under a given condition, and in this situation $\mathrm{SCF}^{\mathrm{FBS}}$ may integrate a response by ubiquitylating two distinct protein types, each interacting with a different region of the FBS substrate adapter. Alternatively, $\mathrm{N}$ - and C-terminal targeting may be asynchronous and condition dependent, in which case $\mathrm{SCF}^{\mathrm{FBS}}$ may entail a switch that works in or leads to two different cellular states. At this point, however, we cannot completely exclude the possibility that FBIPs are not targets (see above), but instead serve in an alternative capacity that enables or inhibits the FBS action. One idea then is that FBIPs are accessories that help recruit other proteins as ubiquitylation targets. In humans, $\mathrm{Cks} 1$ directly associates with the N-terminus of FBX protein Skp2 to direct $\mathrm{SCF}{ }^{S k p 2}$ interaction with ubiquitylation target p27 in human cell cycle regulation $[10,50]$. In Arabidopsis, KAI2 and D14 interact with FBX protein MAX2 in SCF ${ }^{\mathrm{MAX} 2}$ complex to mediate ubiquitylation of SMXL transcription factors [51], though in these cases KAI2 and D14 are also FBX C-terminal interactors. To address these scenarios or others a critical piece of information to learn is the identity of a FBS C-terminal region-interacting protein that we presume to exist. Future work can then investigate higher order $\mathrm{SCF} \mathrm{FBS}^{\mathrm{PB}}$ complex assembly and action.

The 14-3-3 proteins directly regulate a range of cellular processes in plant cells [52], including core signaling pathways and transcriptional reprogramming events in cold and salt stress responses [22-26]. FBS protein interactions with FBIPs will almost certainly be a vital tool used to fully understand the connections between FBS proteins and the 14-3-3 protein regulatory network. Five of 13 Arabidopsis 14-3-3 proteins interact with FBS1. However, 14-3-3 proteins are unlikely ubiquitylation targets of $\mathrm{SCF}^{\mathrm{FBS1}}$ and the consequences of these interactions are unknown [20]. One hypothesis regarding this interaction is that 14-3-3 proteins promote dimerization of $\mathrm{SCF}^{\mathrm{FBS}}$ ligases [20], which in other situations enhances ubiquitylation targeting by SCF complexes $[53,54]$. As our understanding further develops regarding FBIPs as putative targets, their cellular abundance will be an essential readout in studies that investigate 14-3-3 effects on $\mathrm{SCF}^{\mathrm{FBS}}$ activity. The 14-3-3 proteins exert regulatory effects through other mechanisms, however, through controlling the subcellular localization of client proteins or by shifting the location themselves [52]. In salt stress, FBS1 interactors $14-3-3 \lambda$ and $14-3-3 \mathrm{~K}$ act at the plasma membrane and release signaling component SOS2 to activate salt stress tolerance $[26,55]$. Cold temperature triggers the FBS1 interactor 14-3-3 $\lambda$ to translocate from the cytosol into the nucleus where it interacts with and adjusts cold-responsive C-repeat-binding factor (CBF) action [25]. Considering that the FBS1 interaction with FBIPs was exclusively nuclear under the conditions tested here, an investigation of temporal and spatial aspects of 14-3-3/FBS interactions relative to FBS/FBIP interactions in plant cells before and during environmental stress will add more broadly to our understanding of the 14-3-3 stress response network in plant cells.

\section{Materials and Methods}

Bioinformatics: Gene and protein sequences were obtained from The Arabidopsis Information Resource (http:/ / www.arabidopsis.org, accessed on 16 September 2021). Protein sequences were aligned using T-COFFEE (http:/ / www.ebi.ac.uk/Tools/msa/tcoffee, accessed on 16 September 2021) accessed through the European Bioinformatics Institute (EBI) website (http:/ / www.ebi.ac.uk, accessed on 16 September 2021) [56]. WD40 repeat-like sequences were identified in FBIP1 and FBIP2 using the WD40-repeat protein Structures Predictor database version 2.0 (WDSPdb 2.0; http:/ / www.wdspdb.com/wdsp/, accessed on 16 September 2021) [31]. Basic Local Alignment Search Tool (BLAST) and PositionSpecific Iterative (PSI)-BLAST were accessed through the National Center for Biotechnology Information (NCBI) website (http:/ / www.ncbi.nlm.nih.gov, accessed on 16 September 2021) and used to search the RefSeq database. Candidate protein interactors were identified by searching the SUBA4 database (http:/ / suba.live/, accessed on 16 September 2021) [29].

Gateway cloning: Gene-specific primers (Supplementary Table S1) were used with PCR to amplify coding sequences from pooled Arabidopsis thaliana (accession Col-0) cDNA. Amplicons were inserted into the pENTR/D-TOPO vector (Thermo Fisher Scientific, 
Waltham, MA, USA) according to the manufacturer's protocols. Then, the genes were transferred with the LR Clonase II enzyme mix (Thermo Fisher Scientific, Waltham, MA, USA) into pCL112 or pCL113 [57] destination vectors for BiFC experiments, and into pGBKT7-GW (Addgene plasmid \#61703) or pGADT7-GW (Addgene plasmid \#61702) destination vectors for yeast two-hybrid experiments. Alternatively (Figure 3B), FBS1

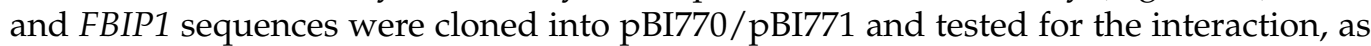
done previously [20]. Primers used to create FBS1 truncation constructs are indicated in Supplementary Table S1.

Yeast two-hybrid assays: Saccharomyces cerevisiae cells were grown, transformed, mated, and selected by standard yeast protocols. Bait constructs (GAL4 DNA-binding domain, DBD) were transformed into Y2H Gold and prey constructs (GAL4 activation domain, AD) and Y187 strains by the LiAc method (Takara Bio; San Jose, CA, USA). Haploid strains were mated to produce diploid strains to test for the interactions. Diploid strains were grown for $24 \mathrm{~h}$ at $30^{\circ} \mathrm{C}$ in the liquid synthetic defined (SD) medium minus Trp/Leu (-TL) medium with shaking. Thereafter, cells were washed in sterile water, cell concentrations were adjusted to $\mathrm{OD}_{600}=10^{0}, 10^{-1}, 10^{-2}, 10^{-3}$, and $10 \mu \mathrm{L}$ was spotted on SD -TL (control), SD minus Trp/Leu/His (-TLH), and SD minus Trp/Leu/His (-TLHA) selective plates. The plates were incubated for 2 days at $30^{\circ} \mathrm{C}$ and then scanned to produce images.

Bimolecular fluorescence complementation (BiFC): Recombinant plasmids were transformed into the Agrobacterium tumefaciens strain GV3101 (pMP90) by electroporation and selected under appropriate antibiotics. A. tumefaciens seed cultures were grown in LB with the appropriate antibiotic selection for 2 days with shaking at $30{ }^{\circ} \mathrm{C}$. Then, they were used to inoculate $50 \mathrm{~mL}$ LB containing the appropriate antibiotics plus $10 \mu \mathrm{M}$ acetosyringone and grown for an additional $24 \mathrm{~h}$. The cells were pelleted and resuspended in the infiltration medium (10 mM MES, $10 \mathrm{mM} \mathrm{MgCl}$, $100 \mu \mathrm{M}$ acetosyringone) and incubated for $5 \mathrm{~h}$ with rocking at room temperature. The cells were pelleted a second time, resuspended in the infiltration medium, and the appropriate nYFP/cYFP, H2B-RFP constructs were combined at a final $\mathrm{OD}_{600}$ of 1.0 for each test/control construct with suppressor strains (p19, $\gamma \beta$, PtoHA, HcPro) at a final $\mathrm{OD}_{600}$ of 0.5. Nicotiana benthamiana leaves from 4-week-old plants were infiltrated by a syringe with the $A$. tumefaciens mixes. The underside of whole leaf mounts was visualized using laser-scanning confocal microscopy 3 days after infiltration with a Nikon D-Eclipse C1 Confocal laser scanning microscope (Nikon Instruments) with either: (1) Excitation at $488 \mathrm{~nm}$ with an emission band pass filter of 515/30 or (2) excitation at $561 \mathrm{~nm}$ with an emission band pass filter of $650 \mathrm{LP}$.

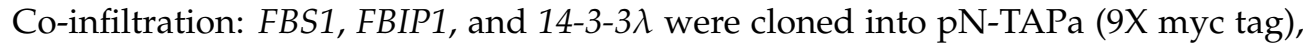
pGWB14 (3X HA tag) or pGWB12 (VSVG tag) vectors [58], respectively, using a Gateway strategy as above. Recombinant plasmids were transformed by electroporation into the A. tumefaciens strain C58C1Rif/pGV2260. A. tumefaciens was grown to a stationary phase in the LB medium containing the appropriate antibiotics plus $50 \mu \mathrm{g} / \mathrm{mL}$ acetosyringone. Bacteria were pelleted and washed with $10 \mathrm{mM} \mathrm{MgCl}_{2}$, and then resuspended in $10 \mathrm{mM}$ $\mathrm{MgCl}_{2}$ and $150 \mu \mathrm{g} / \mathrm{mL}$ acetosyringone. Cell densities were adjusted to $\mathrm{OD}_{600}$ of 0.5 . After $3 \mathrm{~h}$ of incubation, A. tumefaciens strains containing each construct were adjusted to varying concentrations and mixed with the same volume of an $A$. tumefaciens strain containing the viral suppressor $\mathrm{p} 19$, treated in the same way, but adjusted to $\mathrm{OD}_{600}$ of 1.0. The abaxial side of leaves from 3-4 week-old $N$. benthamiana were infiltrated with this bacterial suspension. After 3 days, the leaf material was collected and immediately frozen in liquid $\mathrm{N}_{2}$ for protein extraction.

Protein extraction and Western blotting: Approximately $100 \mu \mathrm{g}$ of frozen tissue was homogenized in $200 \mu \mathrm{L}$ of $1 \times$ Laemmli loading buffer plus $4 \mathrm{M}$ urea, boiled for $5 \mathrm{~min}$, and centrifuged at $10,000 \times g$ for $5 \mathrm{~min}$. Then, $10 \mu \mathrm{L}$ of the supernatant were loaded onto $8 \%, 10 \%$ or $15 \%$ polyacrylamide gels and subjected to SDS-PAGE using the standard protocols. The separated proteins were blotted onto a Hybond-P+ membrane (Amersham Pharmacia Biotech, Amersham, UK) using the standard protocols, and then the membranes were probed with anti-c-Myc, anti-HA antibody or anti-VSVG antibodies (all from Sigma- 
Aldrich, St. Louis, MO, USA). The blots were developed using an alkaline phosphatase kit (BCIP/NBT kit; Invitrogen; Waltham, MA, USA).

AGI numbers: FBS1 (At1g61340), FBS2 (At4g21510), FBS3 (At4g05010), FBS4 (At4g35930), FBIP1 (At3g54190), and FBIP2 (At2g38630).

Supplementary Materials: The following are available online at https: / www.mdpi.com/article / 10.3390/plants10102228/s1, Figure S1: Yeast two-hybrid FBS1-FBS4 interactions with FBIP1 and FBIP2. Figure S2: Bimolecular fluorescence complementation (BiFC) interactions between FBS1-FBS4 and FBIP1. Figure S3: Bimolecular fluorescence complementation (BiFC) interactions between FBS1FBS4 and FBIP2. Figure S4: YFP channel positive and negative controls. Figure S5: FBS1 influence on 14-3-3 $\lambda$ protein abundance in plants. Table S1: Primer sequences used for cloning.

Author Contributions: E.S.-G., E.C.F., E.V.P., L.E.O., A.A.F., A.J.R., M.R.-S., J.M.G. and B.T. designed the experiments. E.S.-G., E.C.F., E.V.P., L.E.O., A.A.F., A.J.R. and B.T. conducted the experiments and analyzed the data. B.T. wrote the manuscript. All authors have read and agreed to the published version of the manuscript.

Funding: This research was funded by grants from the M.J. Murdock Charitable Trust (NS-2016262 and 20141205:MNL:11/20/14) for materials and student summer research stipends, and funds from the University Enrichment Committee (UEC) at the University of Puget Sound for materials and student summer research stipends.

Institutional Review Board Statement: Not applicable.

Informed Consent Statement: Not applicable.

Data Availability Statement: Not applicable.

Acknowledgments: We thank David Somers (The Ohio State University) for the H2B-RFP construct, Frank Harmon (University of California at Berkeley/USDA Plant Gene Expression Center) for the yeast two-hybrid vectors, Faride Unda (University of British Columbia) for the BiFC vectors, and Ruirui Huang and Vivian Irish (Yale University) for the yeast two-hybrid TOPLESS constructs. Moreover, we thank Andreas Madlung (University of Puget Sound) for critical reading of the manuscript and other helpful discussions. Finally, we would like to thank Michal Morrison-Kerr (University of Puget Sound) for her indispensable help in supporting Puget Sound undergraduate research students.

Conflicts of Interest: The authors declare no conflict of interest. The funders had no role in the design of the study; in the collection, analyses, or interpretation of data; in the writing of the manuscript, or in the decision to publish the results.

\section{References}

1. Hua, Z.; Vierstra, R.D. The Cullin-RING Ubiquitin-Protein Ligases. Annu. Rev. Plant Biol. 2011, 62, 299-334. [CrossRef]

2. Gagne, J.M.; Downes, B.P.; Shiu, S.H.; Durski, A.M.; Vierstra, R.D. The F-Box Subunit of the SCF E3 Complex Is Encoded by a Diverse Superfamily of Genes in Arabidopsis. Proc. Natl. Acad. Sci. USA 2002, 99, 11519-11524. [CrossRef]

3. Sheard, L.B.; Tan, X.; Mao, H.; Withers, J.; Ben-Nissan, G.; Hinds, T.R.; Kobayashi, Y.; Hsu, F.F.; Sharon, M.; Browse, J.; et al. Jasmonate Perception by Inositol-Phosphate-Potentiated COI1-JAZ Co-Receptor. Nature 2010, 468, 400-405. [CrossRef]

4. Fang, Q.; Zhou, F.; Zhang, Y.; Singh, S.; Huang, C. Degradation of STOP1 Mediated by the F-box Proteins RAH1 and RAE1 Balances Aluminum Resistance and Plant Growth in Arabidopsis Thaliana. Plant J. 2021, 106, 493-506. [CrossRef] [PubMed]

5. Wang, P.; Nolan, T.M.; Clark, N.M.; Jiang, H.; Montes-Serey, C.; Guo, H.; Bassham, D.C.; Walley, J.W.; Yin, Y. The F-Box E3 Ubiquitin Ligase BAF1 Mediates the Degradation of the Brassinosteroid-Activated Transcription Factor BES1 through Selective Autophagy in Arabidopsis. Plant Cell 2021, koab210. [CrossRef]

6. Jin, J. Systematic Analysis and Nomenclature of Mammalian F-Box Proteins. Genes Dev. 2004, 18, 2573-2580. [CrossRef] [PubMed]

7. Wang, Z.; Liu, P.; Inuzuka, H.; Wei, W. Roles of F-Box Proteins in Cancer. Nat. Rev. Cancer 2014, 14, 233-247. [CrossRef]

8. Lee, C.-M.; Feke, A.; Li, M.-W.; Adamchek, C.; Webb, K.; Pruneda-Paz, J.; Bennett, E.J.; Kay, S.A.; Gendron, J.M. Decoys Untangle Complicated Redundancy and Reveal Targets of Circadian Clock F-Box Proteins. Plant Physiol. 2018, 177, 1170-1186. [CrossRef] [PubMed]

9. Matsumoto, A.; Tateishi, Y.; Onoyama, I.; Okita, Y.; Nakayama, K.; Nakayama, K.I. Fbxw7 $\beta$ Resides in the Endoplasmic Reticulum Membrane and Protects Cells from Oxidative Stress. Cancer Sci. 2011, 102, 749-755. [CrossRef]

10. Spruck, C.; Strohmaier, H.; Watson, M.; Smith, A.P.L.; Ryan, A.; Krek, W.; Reed, S.I. A CDK-Independent Function of Mammalian Cks1: Targeting of SCFSkp2 to the CDK Inhibitor P27Kip. Mol. Cell 2001, 7, 12. [CrossRef] 
11. Kirk, R.; Laman, H.; Knowles, P.P.; Murray-Rust, J.; Lomonosov, M.; Meziane, E.K.; McDonald, N.Q. Structure of a Conserved Dimerization Domain within the F-Box Protein Fbxo7 and the PI31 Proteasome Inhibitor. J. Biol. Chem. 2008, 283, 22325-22335. [CrossRef]

12. Nelson, D.E.; Randle, S.J.; Laman, H. Beyond Ubiquitination: The Atypical Functions of Fbxo7 and Other F-Box Proteins. Open Biol. 2013, 3, 130131. [CrossRef] [PubMed]

13. Kim, W.Y.; Fujiwara, S.; Suh, S.S.; Kim, J.; Kim, Y.; Han, L.; David, K.; Putterill, J.; Nam, H.G.; Somers, D.E. ZEITLUPE Is a Circadian Photoreceptor Stabilized by GIGANTEA in Blue Light. Nature 2007, 449, 356-360. [CrossRef]

14. Sawa, M.; Nusinow, D.A.; Kay, S.A.; Imaizumi, T. FKF1 and GIGANTEA Complex Formation Is Required for Day-Length Measurement in Arabidopsis. Science 2007, 318, 261-265. [CrossRef]

15. Yasuhara, M. Identification of ASK and Clock-Associated Proteins as Molecular Partners of LKP2 (LOV Kelch Protein 2) in Arabidopsis. J. Exp. Bot. 2004, 55, 2015-2027. [CrossRef] [PubMed]

16. Zoltowski, B.D.; Imaizumi, T. Structure and Function of the ZTL/FKF1/LKP2 Group Proteins in Arabidopsis. In The Enzymes; Elsevier: Amsterdam, The Netherlands, 2014; Volume 35, pp. 213-239.

17. Más, P.; Kim, W.-Y.; Somers, D.E.; Kay, S.A. Targeted Degradation of TOC1 by ZTL Modulates Circadian Function in Arabidopsis Thaliana. Nature 2003, 426, 567-570. [CrossRef] [PubMed]

18. Song, Y.H.; Estrada, D.A.; Johnson, R.S.; Kim, S.K.; Lee, S.Y.; MacCoss, M.J.; Imaizumi, T. Distinct Roles of FKF1, GIGANTEA, and ZEITLUPE Proteins in the Regulation of CONSTANS Stability in Arabidopsis Photoperiodic Flowering. Proc. Natl. Acad. Sci. USA 2014, 111, 17672-17677. [CrossRef] [PubMed]

19. Maldonado-Calderon, M.T.; Sepulveda-Garcia, E.; Rocha-Sosa, M. Characterization of Novel F-Box Proteins in Plants Induced by Biotic and Abiotic Stress. Plant Sci. Int. J. Exp. Plant Biol. 2012, 185, 208-217. [CrossRef] [PubMed]

20. Sepulveda-Garcia, E.; Rocha-Sosa, M. The Arabidopsis F-Box Protein AtFBS1 Interacts with 14-3-3 Proteins. Plant Sci. Int. J. Exp. Plant Biol. 2012, 195, 36-47. [CrossRef] [PubMed]

21. Gonzalez, L.E.; Keller, K.; Chan, K.X.; Gessel, M.M.; Thines, B.C. Transcriptome Analysis Uncovers Arabidopsis F-BOX STRESS INDUCED 1 as a Regulator of Jasmonic Acid and Abscisic Acid Stress Gene Expression. BMC Genom. 2017, 18, 533. [CrossRef]

22. Catala, R.; Lopez-Cobollo, R.; Mar Castellano, M.; Angosto, T.; Alonso, J.M.; Ecker, J.R.; Salinas, J. The Arabidopsis 14-3-3 Protein RARE COLD INDUCIBLE 1A Links Low-Temperature Response and Ethylene Biosynthesis to Regulate Freezing Tolerance and Cold Acclimation. Plant Cell 2014, 26, 3326-3342. [CrossRef]

23. Van Kleeff, P.J.; Jaspert, N.; Li, K.W.; Rauch, S.; Oecking, C.; de Boer, A.H. Higher Order Arabidopsis 14-3-3 Mutants Show 14-3-3 Involvement in Primary Root Growth Both under Control and Abiotic Stress Conditions. J. Exp. Bot. 2014, 65, 5877-5888 [CrossRef] [PubMed]

24. Zhou, H.; Lin, H.; Chen, S.; Becker, K.; Yang, Y.; Zhao, J.; Kudla, J.; Schumaker, K.S.; Guo, Y. Inhibition of the Arabidopsis Salt Overly Sensitive Pathway by 14-3-3 Proteins. Plant Cell 2014, 26, 1166-1182. [CrossRef]

25. Liu, Z.; Jia, Y.; Ding, Y.; Shi, Y.; Li, Z.; Guo, Y.; Gong, Z.; Yang, S. Plasma Membrane CRPK1-Mediated Phosphorylation of 14-3-3 Proteins Induces Their Nuclear Import to Fine-Tune CBF Signaling during Cold Response. Mol. Cell 2017, 66, 117-128. [CrossRef] [PubMed]

26. Yang, Z.; Wang, C.; Xue, Y.; Liu, X.; Chen, S.; Song, C.; Yang, Y.; Guo, Y. Calcium-Activated 14-3-3 Proteins as a Molecular Switch in Salt Stress Tolerance. Nat. Commun. 2019, 10, 1199. [CrossRef] [PubMed]

27. Kuroda, H.; Yanagawa, Y.; Takahashi, N.; Horii, Y.; Matsui, M. A Comprehensive Analysis of Interaction and Localization of Arabidopsis SKP1-like (ASK) and F-Box (FBX) Proteins. PLoS ONE 2012, 7, e50009. [CrossRef] [PubMed]

28. Arabidopsis Interactome Mapping Consortium; Dreze, M.; Carvunis, A.-R.; Charloteaux, B.; Galli, M.; Pevzner, S.J.; Tasan, M.; Ahn, Y.-Y.; Balumuri, P.; Barabasi, A.-L.; et al. Evidence for Network Evolution in an Arabidopsis Interactome Map. Science 2011, 333, 601-607. [CrossRef]

29. Hooper, C.M.; Castleden, I.R.; Tanz, S.K.; Aryamanesh, N.; Millar, A.H. SUBA4: The Interactive Data Analysis Centre for Arabidopsis Subcellular Protein Locations. Nucleic Acids Res. 2017, 45, D1064-D1074. [CrossRef]

30. Jain, B.P.; Pandey, S. WD40 Repeat Proteins: Signalling Scaffold with Diverse Functions. Protein J 2018, 37, 391-406. [CrossRef] [PubMed]

31. Ma, J.; An, K.; Zhou, J.-B.; Wu, N.-S.; Wang, Y.; Ye, Z.-Q.; Wu, Y.-D. WDSPdb: An Updated Resource for WD40 Proteins. Bioinformatics 2019, 35, 4824-4826. [CrossRef] [PubMed]

32. Schmid, M.; Davison, T.S.; Henz, S.R.; Pape, U.J.; Demar, M.; Vingron, M.; Scholkopf, B.; Weigel, D.; Lohmann, J.U. A Gene Expression Map of Arabidopsis Thaliana Development. Nat. Genet. 2005, 37, 501-506. [CrossRef]

33. Kilian, J.; Whitehead, D.; Horak, J.; Wanke, D.; Weinl, S.; Batistic, O.; D’Angelo, C.; Bornberg-Bauer, E.; Kudla, J.; Harter, K. The AtGenExpress Global Stress Expression Data Set: Protocols, Evaluation and Model Data Analysis of UV-B Light, Drought and Cold Stress Responses. Plant J. Cell Mol. Biol. 2007, 50, 347-363. [CrossRef] [PubMed]

34. Goda, H.; Sasaki, E.; Akiyama, K.; Maruyama-Nakashita, A.; Nakabayashi, K.; Li, W.; Ogawa, M.; Yamauchi, Y.; Preston, J.; Aoki, K.; et al. The AtGenExpress Hormone and Chemical Treatment Data Set: Experimental Design, Data Evaluation, Model Data Analysis and Data Access. Plant J. 2008, 55, 526-542. [CrossRef] [PubMed]

35. Kagale, S.; Rozwadowski, K. EAR Motif-Mediated Transcriptional Repression in Plants: An Underlying Mechanism for Epigenetic Regulation of Gene Expression. Epigenetics 2011, 6, 141-146. [CrossRef] [PubMed] 
36. Shyu, C.; Figueroa, P.; DePew, C.L.; Cooke, T.F.; Sheard, L.B.; Moreno, J.E.; Katsir, L.; Zheng, N.; Browse, J.; Howe, G.A. JAZ8 Lacks a Canonical Degron and Has an EAR Motif That Mediates Transcriptional Repression of Jasmonate Responses in Arabidopsis. Plant Cell 2012, 24, 536-550. [CrossRef]

37. Long, J.A. TOPLESS Regulates Apical Embryonic Fate in Arabidopsis. Science 2006, 312, 1520-1523. [CrossRef]

38. Pauwels, L.; Barbero, G.F.; Geerinck, J.; Tilleman, S.; Grunewald, W.; Pérez, A.C.; Chico, J.M.; Bossche, R.V.; Sewell, J.; Gil, E.; et al. NINJA Connects the Co-Repressor TOPLESS to Jasmonate Signalling. Nature 2010, 464, 788-791. [CrossRef]

39. Causier, B.; Ashworth, M.; Guo, W.; Davies, B. The TOPLESS Interactome: A Framework for Gene Repression in Arabidopsis. Plant Physiol. 2012, 158, 423-438. [CrossRef]

40. Wang, L.; Kim, J.; Somers, D.E. Transcriptional Corepressor TOPLESS Complexes with Pseudoresponse Regulator Proteins and Histone Deacetylases to Regulate Circadian Transcription. Proc. Natl. Acad. Sci. USA 2013, 110, 761-766. [CrossRef]

41. Dos Santos Maraschin, F.; Memelink, J.; Offringa, R. Auxin-Induced, SCF TIR1-Mediated Poly-Ubiquitination Marks AUX/IAA Proteins for Degradation. Plant J. 2009, 59, 100-109. [CrossRef]

42. Ke, J.; Ma, H.; Gu, X.; Thelen, A.; Brunzelle, J.S.; Li, J.; Xu, H.E.; Melcher, K. Structural Basis for Recognition of Diverse Transcriptional Repressors by the TOPLESS Family of Corepressors. Sci. Adv. 2015, 1, e1500107. [CrossRef]

43. Long, Y.; Schiefelbein, J. Novel TTG1 Mutants Modify Root-Hair Pattern Formation in Arabidopsis. Front. Plant Sci. 2020, 11, 383. [CrossRef] [PubMed]

44. Li, H.; He, Z.; Lu, G.; Lee, S.C.; Alonso, J.; Ecker, J.R.; Luan, S. A WD40 Domain Cyclophilin Interacts with Histone H3 and Functions in Gene Repression and Organogenesis in Arabidopsis. Plant Cell 2007, 19, 2403-2416. [CrossRef] [PubMed]

45. Zhu, J.; Jeong, J.C.; Zhu, Y.; Sokolchik, I.; Miyazaki, S.; Zhu, J.-K.; Hasegawa, P.M.; Bohnert, H.J.; Shi, H.; Yun, D.-J.; et al. Involvement of Arabidopsis HOS15 in Histone Deacetylation and Cold Tolerance. Proc. Natl. Acad. Sci. USA 2008, 105, 4945-4950. [CrossRef] [PubMed]

46. Mehdi, S.; Derkacheva, M.; Ramström, M.; Kralemann, L.; Bergquist, J.; Hennig, L. The WD40 Domain Protein MSI1 Functions in a Histone Deacetylase Complex to Fine-Tune Abscisic Acid Signaling. Plant Cell 2016, 28, 42-54. [CrossRef] [PubMed]

47. Krogan, N.T.; Hogan, K.; Long, J.A. APETALA2 Negatively Regulates Multiple Floral Organ Identity Genes in Arabidopsis by Recruiting the Co-Repressor TOPLESS and the Histone Deacetylase HDA19. Development 2012, 139, 4180-4190. [CrossRef]

48. Leydon, A.R.; Wang, W.; Gala, H.P.; Gilmour, S.; Juarez-Solis, S.; Zahler, M.L.; Zemke, J.E.; Zheng, N.; Nemhauser, J.L. Repression by the Arabidopsis TOPLESS Corepressor Requires Association with the Core Mediator Complex. eLife 2021, 10, e66739. [CrossRef]

49. Lloyd, A.; Brockman, A.; Aguirre, L.; Campbell, A.; Bean, A.; Cantero, A.; Gonzalez, A. Advances in the MYB-BHLH-WD Repeat (MBW) Pigment Regulatory Model: Addition of a WRKY Factor and Co-Option of an Anthocyanin MYB for Betalain Regulation. Plant Cell Physiol. 2017, 58, 1431-1441. [CrossRef]

50. Skaar, J.R.; Pagan, J.K.; Pagano, M. Mechanisms and Function of Substrate Recruitment by F-Box Proteins. Nat. Rev. Mol. Cell Biol. 2013, 14, 369-381. [CrossRef]

51. Wang, L.; Xu, Q.; Yu, H.; Ma, H.; Li, X.; Yang, J.; Chu, J.; Xie, Q.; Wang, Y.; Smith, S.M.; et al. Strigolactone and Karrikin Signaling Pathways Elicit Ubiquitination and Proteolysis of SMXL2 to Regulate Hypocotyl Elongation in Arabidopsis Thaliana. Plant Cell 2020, 32, 2251-2270. [CrossRef]

52. Zhao, X.; Li, F.; Li, K. The 14-3-3 Proteins: Regulators of Plant Metabolism and Stress Responses. Plant Biol. J. 2021, 23, 531-539. [CrossRef]

53. Welcker, M.; Larimore, E.A.; Swanger, J.; Bengoechea-Alonso, M.T.; Grim, J.E.; Ericsson, J.; Zheng, N.; Clurman, B.E. Fbw7 Dimerization Determines the Specificity and Robustness of Substrate Degradation. Genes Dev. 2013, 27, 2531-2536. [CrossRef]

54. Barbash, O.; Lee, E.; Diehl, J. Phosphorylation-Dependent Regulation of SCFFbx4 Dimerization and Activity Involves a Novel Component. Oncogene 2011, 30, 1995-2002. [CrossRef] [PubMed]

55. Tan, T.; Cai, J.; Zhan, E.; Yang, Y.; Zhao, J.; Guo, Y.; Zhou, H. Stability and Localization of 14-3-3 Proteins Are Involved in Salt Tolerance in Arabidopsis. Plant Mol. Biol. 2016, 92, 391-400. [CrossRef] [PubMed]

56. Madeira, F.; mi Park, Y.; Lee, J.; Buso, N.; Gur, T.; Madhusoodanan, N.; Basutkar, P.; Tivey, A.R.N.; Potter, S.C.; Finn, R.D.; et al The EMBL-EBI Search and Sequence Analysis Tools APIs in 2019. Nucleic Acids Res. 2019, 47, W636-W641. [CrossRef] [PubMed]

57. Zhu, D.; Maier, A.; Lee, J.-H.; Laubinger, S.; Saijo, Y.; Wang, H.; Qu, L.-J.; Hoecker, U.; Deng, X.W. Biochemical Characterization of Arabidopsis Complexes Containing constitutively photomorphogenic1 and suppressor of phya Proteins in Light Control of Plant Development. Plant Cell 2008, 20, 2307-2323. [CrossRef]

58. Nakagawa, T.; Kurose, T.; Hino, T.; Tanaka, K.; Kawamukai, M.; Niwa, Y.; Toyooka, K.; Matsuoka, K.; Jinbo, T.; Kimura, T. Development of Series of Gateway Binary Vectors, PGWBs, for Realizing Efficient Construction of Fusion Genes for Plant Transformation. J. Biosci. Bioeng. 2007, 104, 34-41. [CrossRef] [PubMed] 